\title{
Availability Optimization of CO-Shift Conversion System of a Fertilizer Plant using Genetic Algorithm Technique
}

\author{
Sanjeev Kumar, ${ }^{a}$ P. C. Tewari ${ }^{b}$ and Sunand Kuma ${ }^{c}$ and Meenu Guptad \\ ${ }^{a}$ Department of Mechanical Engineering, AKG Engineering College, Ghaziabad - 201009 (Uttar Pradesh), \\ India, ${ }^{b, d}$ Meenu Department of Mechanical Engineering, National Institute of Technology, Kurukshetra - 136119 \\ (Haryana) India, ${ }^{c}$ Department of Mechanical Engineering, National Institute of Technology, Hamirpur - 177005 \\ (Himachal Pradesh) India
}

\begin{abstract}
This paper discusses the availability optimization of CO shift conversion system of a fertilizer plant using Genetic Algorithm. The fertilizer plant comprises of various systems viz. shell gasification and carbon recovery, desulphurization, co-shift conversion, decarbonation- $\mathrm{CO}_{2}$ cooling, $\mathrm{CO}_{2}$ removal, nitrogen wash and ammonia synthesis etc. One of the most important functionaries of a fertilizer plant is CO shift conversion system. The CO shift conversion system of a fertilizer plant has four main subsystems, arranged in series. Considering exponential distribution for the probable failures and repairs, the mathematical formulation of the problem is done using probabilistic approach and differential equations are developed on the basis of Markov birth-death process. These equations are then solved using normalizing conditions so as to determine the steady state availability of the CO shift conversion system. The performance of each subsystem of CO shift conversion system of a fertilizer plant has also been optimized using Genetic Algorithm. So, the findings of the present paper will be highly useful to the plant management for the timely execution of proper maintenance decisions and hence to enhance the system performance.
\end{abstract}

Key words: Performance optimization, CO shift conversion, Genetic algorithm.

\section{Introduction}

Over the years as engineering systems have become more complex and sophisticated, the availability optimization of engineering systems is becoming increasingly important because of factors such as cost, risk of hazard, competition, public demand, usage of new technology. High reliability level is desirable to reduce overall costs of production and risk of hazards for larger, more complex and sophisticated systems such as fertilizer plant. During the last three decades reliability technology has been developed for use in various technological fields (Billinton and Allan, 1983). The technology is mainly used in the development of electrical and electronics equipments. The technology has also been used in a number of industrial and transportation problems.

A fertilizer plant is a complex engineering system comprising of various systems: shell gasification and carbon recovery, desulphurization, co-shift conversion, decarbonation, nitrogen wash and ammonia synthesis etc (Barbady and Kumar, 2008) and (Kumar et. al., 2007). The optimization of each system in relation to one another is imperative to make the plant profitable and viable for operation. Effectiveness of

\footnotetext{
* Corresponding author E-mail: getsan25@rediffmail.com
}

fertilizer plant is mainly influenced by the availability, reliability and maintainability of the plant, and its capability to perform as expected (Kurien, 1988). Reliability analysis techniques have been gradually accepted as standard tools for the planning and operation of automatic and complex fertilizer plants.

Now a days, reliability analysis has become an integral part of system design. This is especially true for systems performing critical applications. System designers rely on commercially available dependability tools in order to assess the reliability of their systems. The usage of such tools becomes therefore crucial. Systems being built are increasingly complex and large; their components are exhibiting behaviors and interactions that are becoming more and more difficult to model and analyze using existing conventional tools. Markov Chains (MCs) and their extensions have proven to be a versatile tool for modeling complex dynamic component behavior. They have been extensively used for dependability analysis of dynamic systems and many tools have adopted, directly or indirectly, MCs as their formalism 
(Butler 1986). During the past decade a lot of study has been done by analysis tools for reliability, availability, performance modeling (Koren and Gaertner, 1987) and (Dhillon and Singh 1981) and (Srinath, 1994).

In the present article, availability of a critical CO- shift conversion system in the fertilizer plant has been worked out using a Markovian model. The need and the relevance for carrying out such a study have been described in the script. The actual failure and repair data on the identified CO shift conversion system has been used in the analysis (Kumar et. al., 1988). The aim of this paper is to present a procedure of availability optimization. Availability has a wider scope than reliability, as it takes into account maintenance time analysis in addition to failure time analysis. The procedure is based on Genetic Algorithm, which is suitable for problems with this degree of complexity. A Genetic Algorithm is a search strategy that employs random choice to guide a highly exploitative search, striking a balance between exploration of the feasible domain and exploitation of 'good' solutions. This strategy is analogous to biological evolution. Different to the classic optimization algorithms, the Genetic Algorithm does not work with only one point in the search space, but with a group of points simultaneously. The number of points is previously determined by a parameter known as population size. The Genetic Algorithm does not need to use differential calculus. It can be considered a robust method, because it is not influenced by local maximum or minimum, discontinuity or noise in the objective function. Genetic Algorithm operators are the instruments used by the algorithm to reach the optimum point of the function. Four operators, described by (Goldberg, 2003), were developed in the computer program: mutation, crossover, population size and generation size. This paper discusses the availability optimization of CO shift conversion in a medium sized fertilizer plant.

\section{System description}

The CO shift conversion system comprises of four subsystems arranged in series:

1. Subsystem (A): Consists of two units in series (humidifier and dehumidifier pump). A cold standby pump is provided with each unit. Complete failure occurs only when standby pump of a unit also fails.

2. Subsystem (B): Consists of two condensate pumps in series. Failure of any unit will cause complete failure of the system.
3. Subsystem (C): Consists of four gas heaters (two working in series and the other two in cold standby). Complete failure occurs only when three heaters are in failed state.

4. Subsystem (D): Consists of nine units in series (humidifier, shift converter, dehumidifier, feed water heater, absorption refrigerator, separators, low pressure boiler, economizer and cooler). Failure of any unit will cause complete failure of the system.

\section{Notations}

The notations used to represent the various states of the subsystems in the transition diagram of CO shift conversion system (Figure 1) are as follows (Kumar et. al. 1988) and (Kumar and Pandey, 1993):

A, B, C, D : denotes that the subsystems are full operating state.

$\mathrm{A}^{1}, \mathrm{C}^{1}, \mathrm{C}^{2} \quad$ : denotes that the subsystems $\mathrm{A}$ and $\mathrm{C}$ are working on standby unit.

a, b, c, d : denotes that the subsystems are in failed state.

$P_{0}(t) \quad$ : probability that at time $t$ all subsystems are in original working state (without standby unit).

$P_{i}(t) \quad: \quad$ probability that at time $t$ all subsystems are in full load condition (standby mode) for $\mathrm{i}$ $=1-5$.

$P_{j}(t) \quad: \quad$ probability that at time $t$ all subsystems are in breakdown state for $\mathrm{j}=6-22$.

$\alpha_{i}, \mathrm{i}=1-4:$ mean failure rates in A, B, C, D.

$\beta_{i}, \mathrm{i}=1-4 \quad$ : mean rate of repairs in A, B, C, D.

$\Delta t \quad$ : time increment

$P_{i} \quad: \quad$ represents derivative w.r.t. 't'.

\section{Assumptions}

The analysis presented herein is based on the following assumptions (Kumar et. al. 1988 and Kumar et. al. 1988):

1. Failure/repair rates are constant over time and statistically independent.

2. A repaired unit is as good as new, performance wise for a specified duration.

3. Sufficient repair facilities are provided as and when required. 
4. Standby units are of the same nature as that of active units.

5. System failure/repair follows exponential distribution.

6. Service includes repair and/or replacement.

7. System may work at reduced capacity.

8. There are no simultaneous failures.

\section{Mathematical Modeling}

Mathematical modeling has been developed for the prediction of steady state availability of the individual components as well as entire system. The failure and repair rates of the different subsystems, available from the maintenance sheets of the fertilizer plant, are used as standard input information for the analysis (Shooman, 1996). The state of the system defines the condition at any instant of time and the information is useful in analyzing the current state and in the prediction of the failure state of the system. If the state of the system is probability based, then the model is a Markov probability model. Markov model is defined by a set of probabilities $P_{i j}$, where $P_{i j}$ is the probability of transition from any state $\mathrm{i}$ to any state $\mathrm{j}$. One of the most important features of the Markov process is that the transition probability $P_{i j}$; depends only on states $i$ and $j$ and is completely independent of all past states except the last one, state i (Kumar et. al., 1999) and (Tewari et al., 2000).

Let the probability of $\mathrm{n}$ occurrences in time $\mathrm{t}$ be denoted by $P_{n}(t)$, i.e.,

Probability $(\mathrm{X}=\mathrm{n}, \mathrm{t})=P_{n}(t) \quad(\mathrm{n}=0,1,2 \ldots)$.

Then, $P_{0}(t)$ represent the probability of zero occurrences in time $\mathrm{t}$. The probability of zero occurrences in time $(\mathrm{t}+\Delta \zeta \mathrm{t})$ is given by equation (1); i.e.

$P_{0}(t+\Delta t)=(1-\beta \mathrm{t}) \cdot P_{0}(t)$

Similarly $P_{1}(t+\Delta t)=(\alpha \cdot \Delta t) \cdot P_{0}(t)+(1-\beta \cdot \Delta t) \cdot P_{1}(t)$

The equation 2 shows the probability of one occurrence in time $(t+\Delta t)$ and is composed of two parts, namely, (a) probability of zero occurrences in time $t$ multiplied by the probability of one occurrence in the interval $\Delta \mathrm{t}$ and (b) the probability of one occurrence in time t multiplied by the probability of no occurrences in the interval $\Delta \mathrm{t}$. Then simplifying and putting $\Delta \mathrm{t} \rightarrow 0$, one gets (Kumar et. al., 2007 and Khanduja et. al., 2008)

$$
\left(\frac{d}{d t}+\alpha\right) P_{1}(t)=\beta P_{0}(t)
$$

Using the concept used in equation 3 and various probability considerations, the following differential equations associated with the transition diagram of CO shift conversion system are formed (Tewari et al, 2000 and Kumar et. al., 2008):

$$
\begin{aligned}
& P_{0}^{\prime}(t)+\left(\sum_{i=1}^{4} \alpha_{i}\right) P_{0}(t)=\beta_{1} P_{1}(t)+\beta_{2} P_{6}(t) \\
& +\beta_{3} P_{2}(t)+\beta_{4} P_{7}(t) \\
& P_{1}^{\prime}(t)+\left(\sum_{i=1}^{4} \alpha_{i}+\beta_{1}\right) P_{1}(t)=\beta_{1} P_{8}(t) \\
& +\beta_{2} P_{9}(t)+\beta_{3} P_{3}(t)+\beta_{4} P_{10}(t)+\alpha_{1} P_{0}(t) \\
& P_{2}^{\prime}(t)+\left(\sum_{i=1}^{4} \alpha_{i}+\beta_{3}\right) P_{2}(t)=\beta_{1} P_{3}(t) \\
& +\beta_{2} P_{11}(t)+\beta_{3} P_{4}(t)+\beta_{4} P_{12}(t)+\alpha_{3} P_{0}(t) \\
& P_{3}^{\prime}(t)+\left(\sum_{i=1}^{4} \alpha_{i}+\beta_{1}+\beta_{3}\right) P_{3}(t)=\sum_{i=1}^{2} \beta_{i} P_{i+12}(t) \\
& +\beta_{3} P_{5}(t)+\beta_{4} P_{15}(t)+\alpha_{1} P_{2}(t)+\alpha_{3} P_{1}(t) \\
& P_{4}^{\prime}(t)+\left(\sum_{i=1}^{4} \alpha_{i}+\beta_{3}\right) P_{4}(t)=\beta_{1} P_{5}(t)+\beta_{2} P_{14}(t) \\
& +\sum_{i=3}^{4} \beta_{i} P_{i+14}(t)+\alpha_{3} P_{2}(t) \\
& P_{5}^{\prime}(t)+\left(\sum_{i=1}^{4} \alpha_{i}+\beta_{3}+\beta_{1}\right) P_{5}(t)=\sum_{i=1}^{4} \beta_{i} P_{i+18}(t) \\
& +\alpha_{3} P_{3}(t)+\alpha_{1} P_{4}(t) \\
& P_{i}^{\prime}(t)+\beta_{m} P_{i}(t)=\alpha_{m} P_{j}(t)
\end{aligned}
$$

With the initial conditions at time $t=0$ are

$P_{i}(t)$ for $t=0$, otherwise $P_{i}(t)=0$.

Since any fertilizer plant is a process industry where raw material is processed through various subsystems continuously till the final product is obtained. Thus, long run availability of the CO shift conversion system is computed by substituting $d / d t=0$ as $t \rightarrow \infty$ in equations (4) to (10). Steady state probabilities for $\mathrm{CO}$ shift conversion system are given as (Kumar et al. 2008):

$$
\begin{aligned}
& P_{i}=\alpha_{m} / \beta_{m} P_{j} \\
& P_{6}=\left(\alpha_{2} / \beta_{2}\right) P_{0} \\
& P_{7}=\left(\alpha_{4} / \beta_{4}\right) P_{0} \\
& P_{8}=\left(\alpha_{1} / \beta_{1}\right) P_{1}
\end{aligned}
$$




$$
\begin{aligned}
& P_{10}=\left(\alpha_{4} / \beta_{4}\right) P_{1} \\
& P_{11}=\left(\alpha_{2} / \beta_{2}\right) P_{2} \\
& P_{12}=\left(\alpha_{4} / \beta_{4}\right) P_{2} \\
& P_{13}=\left(\alpha_{1} / \beta_{1}\right) P_{3} \\
& P_{14}=\left(\alpha_{2} / \beta_{2}\right) P_{3} \\
& P_{15}=\left(\alpha_{4} / \beta_{4}\right) P_{3} \\
& P_{16}=\left(\alpha_{2} / \beta_{2}\right) P_{4} \\
& P_{17}=\left(\alpha_{3} / \beta_{3}\right) P_{4} \\
& P_{18}=\left(\alpha_{4} / \beta_{4}\right) P_{4} \\
& P_{19}=\left(\alpha_{1} / \beta_{1}\right) P_{5} \\
& P_{20}=\left(\alpha_{2} / \beta_{2}\right) P_{5} \\
& P_{21}=\left(\alpha_{3} / \beta_{3}\right) P_{5} \\
& P_{22}=\left(\alpha_{4} / \beta_{4}\right) P_{5}
\end{aligned}
$$

Now putting the values of probabilities from equation 12-28 in equations 4-9, and solving these equations recursively, we get the steady state probabilities (Arora and Kumar, 1997):

$\left(\alpha_{1}+\alpha_{3}\right) P_{0}=\beta_{1} P_{1}+\beta_{3} P_{2}$

$\left(\alpha_{3}+\beta_{1}\right) P_{1}=\beta_{3} P_{3}+\alpha_{1} P_{0}$

$\left(\alpha_{1}+\alpha_{3}+\beta_{3}\right) P_{2}=\beta_{1} P_{3}+\beta_{3} P_{4}+\alpha_{3} P_{0}$

$\left(\alpha_{3}+\beta_{1}+\beta_{3}\right) P_{3}=\beta_{3} P_{5}+\alpha_{1} P_{2}+\alpha_{3} P_{1}$

$\left(\beta_{3}+\alpha_{1}\right) P_{4}=\beta_{1} P_{5}+\alpha_{3} P_{2}$

$\left(\beta_{1}+\beta_{3}\right) P_{5}=\alpha_{1} P_{4}+\alpha_{3} P_{3}$

Mathematical task is performed to solve the set of equations (29)-(34) simultaneously in terms of $P_{0}$ as given by:

$$
\begin{gathered}
\sum_{i=0}^{22} P_{i}=1 \quad P_{0}=N^{-1} \\
N=\left\{\begin{array}{l}
\left\{\begin{array}{l}
\left.1+\left[\left(C_{4} \beta_{3}+\alpha_{1}\right) /\left(\alpha_{3}+\beta_{1}\right)\right]+C_{4}+C_{5}+\left[\left(\beta_{3}+\alpha_{3}+\alpha_{1}\right) C_{5}-\alpha_{3}-\beta_{1} C_{4}\right] / \beta_{3}\right]\left\{1+\frac{\alpha_{2}}{\beta_{2}}+\frac{\alpha_{4}}{\beta_{4}}\right\} \\
{\left[1+\left(\beta_{3}+\alpha_{1}\right)\right] 1 / \beta_{1}-\alpha_{3} C_{5} / \beta_{1}}
\end{array}\right. \\
\left.+\frac{\alpha_{1}}{\beta_{1}}\left\{\left(C_{4} \beta_{3}+\alpha_{1}\right) /\left(\alpha_{3}+\beta_{1}\right)\right]+C_{4}+\left[\left(\beta_{3}+\alpha_{1}\right)\left\{\left(\beta_{3}+\alpha_{3}+\alpha_{1}\right) C_{5}-\alpha_{3}-\beta_{1} C_{4}\right\} 1 / \beta_{3}-\alpha_{3} C_{5}\right] / \beta_{1}\right\} \\
\left.+\frac{\alpha_{3}}{\beta_{3}}\left\{\left[\left(\beta_{3}+\alpha_{3}+\alpha_{1}\right) C_{5}-\alpha_{3}-\beta_{1} C_{4}\right] 1 / \beta_{3}\right]\left(1+\left(\beta_{3}+\alpha_{1}\right) 1 / \beta_{1}\right)-\alpha_{3} C_{5} / \beta_{1}\right\}
\end{array}\right]
\end{gathered}
$$

Where

$$
\begin{aligned}
& C_{1}=1-\frac{\alpha_{1} \beta_{1}}{\alpha_{3} \beta_{3}}\left(1-\left(\alpha_{1}+\alpha_{3}+\beta_{3}\right) /\left(\beta_{1}+\beta_{3}\right)\right) ; \\
& C_{2}=\frac{\alpha_{3}{ }^{2}\left(\beta_{1}+\beta_{3}\right)+\alpha_{3} \beta_{3}\left(2 \beta_{1}+\beta_{3}-1\right)+\alpha_{3} \beta_{1}\left(\beta_{1}+\alpha_{1}\right)}{\alpha_{3}{ }^{2}\left(\beta_{1}+\beta_{3}\right)} \\
& C_{3}=\frac{\alpha_{1}}{\alpha_{3} \beta_{3}}\left(\alpha_{1}+\alpha_{3}\right)+\frac{\alpha_{1}}{\beta_{1}+\beta_{3}}\left(1+\left[\left(\alpha_{3}+\alpha_{1}+\beta_{3}\right) \mid\right.\right. \\
& \left.\left.\left(\alpha_{1}+\alpha_{3}\right)\right] /\left(\alpha_{3} \beta_{3}\right)\right) \\
& C_{4}=\frac{\alpha_{1} C_{1}+C_{3}\left(\alpha_{3}+\beta_{1}\right)}{C_{2}\left(\alpha_{3}+\beta_{1}\right)-\beta_{3} C_{1}} ; \\
& C_{5}=\frac{1}{\beta_{3}}\left\{\left(\alpha_{1}+\alpha_{3}\right)-\beta_{1}\left[\left(C_{4} \beta_{3}+\alpha_{1}\right) /\left(\alpha_{3}+\beta_{1}\right)\right]\right\}
\end{aligned}
$$

The probability of full working capacity, namely, $\mathrm{P}_{0}$ is determined by using normalizing condition: (i.e. sum of the probabilities of all working states, reduced capacity and failed states is equal to 1) (Kumar et. al., 2009): 
Now, the steady state availability of Co shift conversion system may be obtained as summation of all working state probabilities as

Steady state availabil

$$
\begin{aligned}
& (A v .)=\sum_{i=0}^{5} P_{i}=P_{0}+P_{1}+P_{2}+P_{3}+P_{4}+P_{5} \quad \begin{array}{r}
\text { The process is repeated until } \\
\text { same size as the population. }
\end{array} \\
& A v .=\left\{\begin{array}{l}
1+\left(C_{4} \beta_{3}+\alpha_{1}\right) /\left(\alpha_{3}+\beta_{1}\right)+C_{5}+C_{4}+\left[\left(\beta_{3}+\alpha_{3}+\alpha_{1}\right) C_{5}-\alpha_{3}-\beta_{1} C_{4}\right] \frac{1}{\beta_{3}}+ \\
\left.\left\{\beta_{3} C_{5}\left(\beta_{3}+2 \alpha_{1}\right)+\alpha_{1} C_{5}\left(\alpha_{1}+\alpha_{3}\right)-\left(\beta_{3}+\alpha_{1}\right)\left(\beta_{1} C_{4}+\alpha_{3}\right)\right] / \beta_{1} \beta_{3}\right\}
\end{array}\right\} N^{-1}
\end{aligned}
$$

7. The process is repeated until the mating pool has the

ments, consisting of four population members per tournament.

6. Copy the most fit population member in each tournament to the mating pool.
Therefore, availability (Av.) represents the model for steady state availability of CO- shift conversion system. This model includes all possible states of nature, that is, future events $\left(\alpha_{l}\right)$ and the identification of all the courses of action, that is, repair priorities $\left(\beta_{\imath}\right)$. This model is used to implement the maintenance policies for a CO- shift conversion system of a fertilizer plant. The various availability levels may be computed for different combinations of failure and repair rates / priorities. It can be used for availability optimization of this operating system of a fertilizer plant by using Genetic Algorithm Technique.

\section{Genetic Algorithm Technique (G.A.T)}

The Genetic Algorithm is a search method based on concepts of biological evolution and reproduction (Dhillon and Shing 1981). Previous works indicate that a Genetic Algorithm is recommended for problems involving complex mathematical expressions in their modeling. An important advantage is that it does not require the use of differential calculus. The Genetic Algorithms is a model of machine learning, which derives it's behavior from a metaphor of some of the mechanisms of the evolution in the nature (Goldberg, 2003). The action of G.A.T. for parameter optimization in the present problem can be stated as follows:

1. Initialize the parameters of the G.A.T.

2. Randomly generate the initial population and prepare the coded strings.

3. Compute the fitness of each individual in the old population.

4. Form the mating pool from the old population.

5. Divide the population repeatedly into random tourna-
8. Determine the new generation pool.

9. Select two parents from the mating pool randomly.

10. Perform the crossover of the parents to produce to produce two off springs.

11. Mutate, if required.

12. Place the child strings to new population.

13. Compute the fitness of each individual in new population.

14. Replace the old population with the new population.

15. Repeat the steps 3 to 8 until the best individuals in new population represent the optimum value of the performance function (system availability).

\section{Availability optimization using G.A.T.}

The performance behavior of CO- shift conversion system is highly influenced by the failure and repair parameters of each subsystem. These parameters ensure high performance of the CO- shift conversion system. G.A.T. is hereby proposed to coordinate the failure and repair parameters of each subsystem for stable system performance i.e. high availability. Here, number of parameters is eight (four failure parameters and four repair parameters). The design procedure is described as follows (Tsai et. al., 2001):

To use G.A.T. for solving the given problem, the chromosomes are to be coded in real structures. Here, concatenated, multi-parameter, mapped, fixed point coding is used. Unlike, unsigned fixed point integer coding parameters are mapped to a specified interval [Xmin, Xmax], where Xmin and Xmax are the maximum and minimum values of system 
parameters . The maximum value of the availability function corresponds to optimum values of system parameters. These parameters are optimized according to the performance index i.e. desired availability level. To test the proposed method, failure and repair rates are determined simultaneously for optimal value of system availability.

\section{Results and Discussion}

Effects of population size and number of generations on the availability of CO shift conversion system are shown in Table I and II. To specify the computed simulation more precisely, trial sets are also chosen for G.A. and system parameters. The availability of CO- shift conversion system is determined by using the designed values of the system parameters.
Here, real-coded structures are used.

Maximum number of population size is varied from 20 to 160.

Number of generations - 100

Crossover probability $\quad-\quad 0.8$

Mutation probability $\quad-\quad 0.1$

Total number of run $\quad-\quad 01$

The effect of population size on availability of COshift conversion system is shown in Figure 2. The optimum value of system's performance is $92.20 \%$, for which the best possible combination of failure and repair rates is $=0.0021$, $=0.0699,=0.0052,=0.6795,=0.0803,=0.2497,=$

Table I. Effect of population size on availability of CO- shift conversion system

\begin{tabular}{l|c|c|c|c|c|c|c|c|c}
\hline Pop. size & Av. & $\alpha_{1}$ & $\alpha_{2}$ & $\alpha_{3}$ & $\alpha_{4}$ & $\beta_{1}$ & $\beta_{2}$ & $\beta_{3}$ & $\beta_{4}$ \\
\hline 20 & 0.9082 & 0.0021 & 0.0057 & 0.0802 & 0.0024 & 0.0654 & 0.6685 & 0.2387 & 0.0812 \\
40 & 0.9190 & 0.0024 & 0.0052 & 0.0807 & 0.0021 & 0.0689 & 0.6608 & 0.2491 & 0.0998 \\
60 & 0.9134 & 0.0023 & 0.0055 & 0.0827 & 0.0023 & 0.0676 & 0.6416 & 0.2479 & 0.0971 \\
80 & 0.9147 & 0.0022 & 0.0054 & 0.0807 & 0.0022 & 0.0691 & 0.6350 & 0.2431 & 0.0925 \\
100 & 0.9183 & 0.0022 & 0.0050 & 0.0804 & 0.0023 & 0.0694 & 0.6946 & 0.2482 & 0.0992 \\
120 & 0.9207 & 0.0022 & 0.0053 & 0.0803 & 0.0021 & 0.0696 & 0.6661 & 0.2495 & 0.0972 \\
140 & 0.9220 & 0.0021 & 0.0052 & 0.0803 & 0.0020 & 0.0699 & 0.6795 & 0.2497 & 0.0971 \\
160 & 0.9200 & 0.0021 & 0.0050 & 0.0801 & 0.0021 & 0.0651 & 0.6955 & 0.2457 & 0.0995 \\
\hline
\end{tabular}

Table II. Effect of Number of Generations on Availability of CO- Shift Conversion System

\begin{tabular}{l|c|c|c|c|c|c|c|c|c}
\hline Gen. Size & Av. & $\alpha_{1}$ & $\alpha_{2}$ & $\alpha_{3}$ & $\alpha_{4}$ & $\beta_{1}$ & $\beta_{2}$ & $\beta_{3}$ & $\beta_{4}$ \\
\hline 50 & 0.9156 & 0.0024 & 0.0068 & 0.0804 & 0.0022 & 0.0689 & 0.6433 & 0.2493 & 0.0967 \\
100 & 0.9183 & 0.0022 & 0.0050 & 0.0804 & 0.0023 & 0.0694 & 0.6946 & 0.2482 & 0.0992 \\
125 & 0.9193 & 0.0023 & 0.0054 & 0.0804 & 0.0021 & 0.0672 & 0.6560 & 0.2482 & 0.0997 \\
225 & 0.9221 & 0.0021 & 0.0053 & 0.0801 & 0.0020 & 0.0681 & 0.6754 & 0.2499 & 0.0999 \\
255 & 0.9224 & 0.0020 & 0.0053 & 0.0803 & 0.0020 & 0.0692 & 0.6905 & 0.2499 & 0.0993 \\
280 & 0.9220 & 0.0020 & 0.0052 & 0.0804 & 0.0020 & 0.0695 & 0.6844 & 0.2489 & 0.0993 \\
300 & 0.9208 & 0.0022 & 0.0054 & 0.0800 & 0.0021 & 0.0683 & 0.6857 & 0.2495 & 0.0996 \\
\hline
\end{tabular}

Failure and repair rate parameter constraints

$$
\begin{array}{lc}
\left(\alpha_{1}, \beta_{1}, \quad \alpha_{2}, \beta_{2},\right. & \left.\alpha_{3}, \beta_{3}, \alpha_{4}, \beta_{4}\right) \\
\alpha_{1} \varepsilon[0.002,0.008] & \alpha_{2} \varepsilon[0.005,0.02] \\
\alpha_{3} \varepsilon[0.08,0.095] & \alpha_{4} \varepsilon[0.002,0.008] \\
\beta_{1} \varepsilon[0.01,0.07] & \beta_{2} \varepsilon[0.1,0.7] \\
\beta_{3} \varepsilon[0.1,0.25] & \beta_{4} \varepsilon[0.04,0.1]
\end{array}
$$

0.0020, $=0.0971$ at population size 140, as given in Table I.

Maximum number of generations is varied from 50 to 300.

Population size $\quad-100$

Crossover probability $\quad-\quad 0.8$

Mutation probability $\quad-\quad 0.2$

Total number of run $\quad-\quad 01$ 


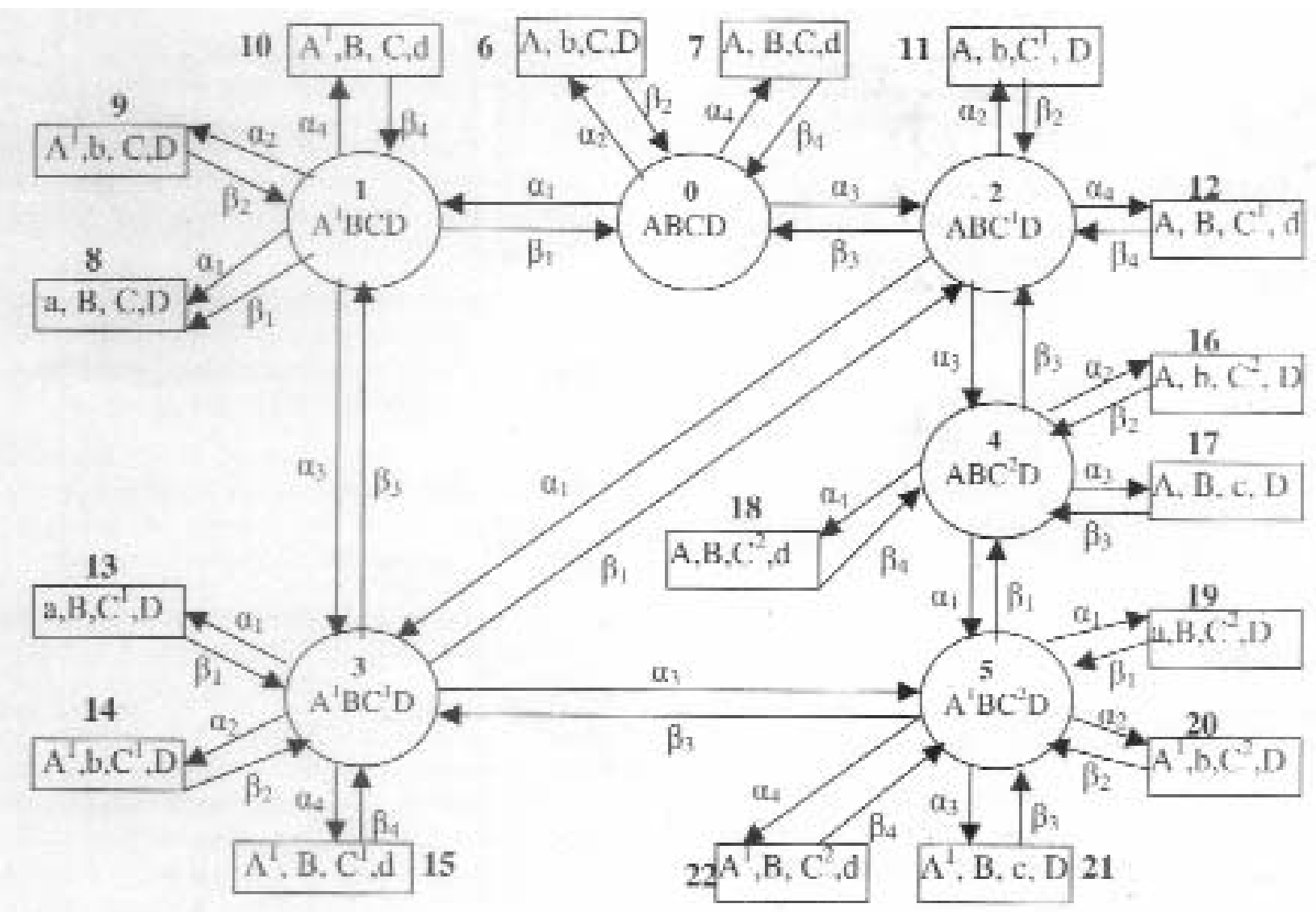

Fig. 1. Transition diagram of CO shift conversion system

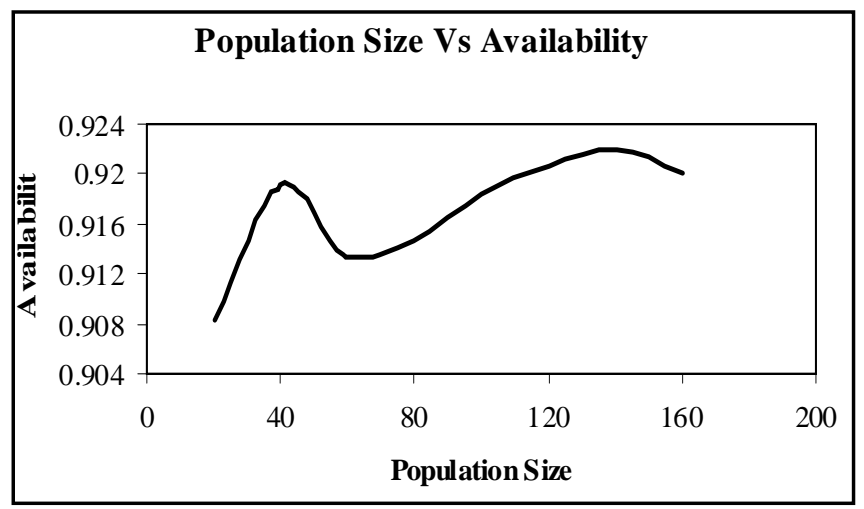

Fig. 2. Effect of population size on availability of the CO- shift conversion system

The effect of number of generations on availability of the CO- shift conversion system is shown in figure 3 . The optimum value of system's performance is $92.24 \%$, for which the best possible combination of failure and repair rates is = $0.0020,=0.0692,=0.0053,=0.6905,=0.0803,=$ 0.2499 , $=0.0020,=0.0993$ at generation size 255 , as given in Table II.

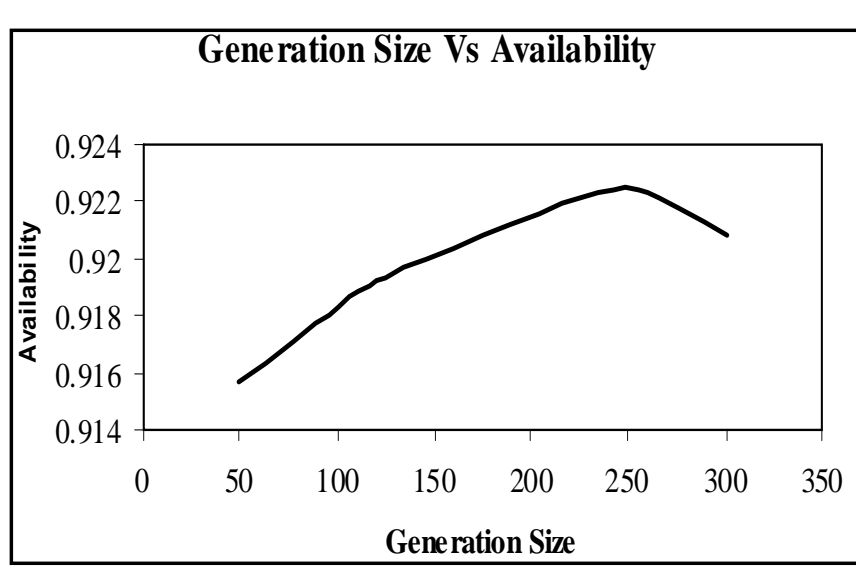

Fig. 3. Effect of number of generations on availability of $\mathrm{CO}$ - shift conversion system

\section{Conclusion}

The availability optimization of the CO- shift conversion system of a fertilizer plant is discussed in this paper. Genetic Algorithm Technique (G.A.T) is hereby proposed to select the various feasible values of the system failure and repair 
parameters. Then, G.A.T is successfully applied to coordinate simultaneously all these parameters for an optimum level of system performance. Besides, the effect of G.A. parameters such as population size and number of generations on system performance i.e. availability has also been discussed. The findings of this paper are discussed with the concerned fertilizer plant management. Such results are found highly beneficial for the purpose of performance optimization of the CO- shift conversion system of a fertilizer plant concerned.

\section{Acknowledgement}

The author is grateful to Sh. Sudesh Chohan, National Fertilizer Limited Panipat (India), for providing every possible help during the work.

\section{References}

Arora N. and Kumar D. (1997) Availability Analysis of Steam and Power Generation Systems in Thermal Power Plant. Microelectronics Reliability 37: 795-799.

Barabady J. and Kumar U. (2008) Reliability analysis of mining equipment: a case study of a crushing. Reliability Engineering and System Safety. 93: 647-653.

Billinton R. and Allan R. N. (1983) Reliability Evaluation of Engineering Systems, Concepts and Techniques, (Pitman Books Limited, Boston), 1983.

Butler R. (1986) The SURE Reliability Analysis Program. AIAA Guidance, Navigation, and Control Conference.

Dhillon B. S. and Singh C. (1981) Engineering ReliabilityNew techniques and applications. (John willey and sons, New York) 1981.

Goldberg D. E. (2003) Genetic Algorithm in SearchOptimization and Machine Learning. (Pearson Education Asia, New Delhi) 2003.

Khanduja R., Tewari P. C. and Kumar D. (2008) Availability Analysis of Bleaching System of Paper Plant. Journal of Industrial Engineering, Udyog Pragati, N.I.T.I.E. Mumbai (India). 32: 24-29.

Koren J. M. and Gaertner J. (1987) CAFTA: A Fault tree analysis tool designed for PSA. Proceedings of Probabilistic Safety Assessment and Risk Management. 2: 588-592.

Kumar D., Singh I. P. and Singh J. (1988) Reliability Analysis of the Feeding System in the Paper Industry. Microelectronics Reliability. 28 (3): 213-215.

Kumar D., Singh I. P. and Singh J. (1988) Availability of the
Feeding System in the Sugar Industry. Microelectronics Reliability. 28 (3): 867- 871.

Kumar D. and Pandey P. C. (1993) Maintenance Planning and Resource Allocation in Urea Fertilizer Plant. International Journal of Quality and Reliability Engineering. 9: 411- 423.

Kumar S., Kumar D. and Mehta N. P. (1999) Maintenance Management for Ammonia Synthesis System in a Urea Fertilizer Plant. International Journal of Management and System (IJOMAS). 15: 211-214.

Kumar S., Tewari P. C.and Sunand K. (2007) Performance Modeling and Simulation of Urea Synthesis System of a Fertilizer Plant. Proceedings of International Conference held at Mumbai (India). pp 645-650.

Kumar S., Tewari P. C. and Sharma R. (2007) Simulated Availability of $\mathrm{CO}_{2}$ Cooling System in a Fertilizer Plant. Industrial Engineering Journal (Indian Institution of Industrial Engineering, Mumbai). 36: 19-23.

Kumar S., Tewari P. C. and Kumar S. (2008) Development of Performance Evaluating Model for CO- Shift Conversion System in the Fertilizer Plant. International Journal of Engineering Research and Industrial Applications (IJERIA).1 (6): 369-382.

Kumar S., Tewari P. C. and Kumar S. (2009) Simulation Model for Evaluating the Performance of Urea Decomposition System in a Fertilizer Plant. International Journal of Industrial Engineering and Practices (I. J. I. E. P.). 1(1):10-14.

Kurien K. C. (1988) Reliability and availability analysis of repairable system using discrete event simulation, $\mathrm{Ph}$. D. Thesis, IIT, New Delhi.

Shooman M. L. (1996) Reliability Computation for Systems with Dependents Failures. Proceedings of IEEE Annual Symposium on Reliability. pp 44-56.

Srinath L. S. (1994) Reliability Engineering. Third edition (East-West Press Pvt. Ltd., New Delhi India) 1994.

Tewari P. C., Kumar D. and Mehta N. P. (2000) Decision Support System of Refining System of Sugar Plant. Journal of Institution of Engineers (India). 84: 41-44.

Tsai Y. T., Wang K. S. and Teng H. Y. (2001) Optimizing preventive maintenance for mechanical components using genetic algorithms. Reliability Engineering and System Safety. 74(1): 89-97.

Received : May 25, 2009;

Accepted : August 24, 2009 